\title{
Fuentes de información en medicina basada en la evidencia
}

\author{
Por José Antonio Salvador Oliván, José María Angós Ullate, Mª Jesús Fernández Ruiz \\ y Rosario Arquero Avilés
}

\begin{abstract}
Resumen: La medicina basada en la evidencia $(M B E)$ ha supuesto un cambio importante en el papel y en el uso de la información por parte del profesional de la salud que debe tomar decisiones apropiadas en su práctica clínica diaria. La MBE ha impulsado la creación de recursos de información propios caracterizados por evaluar, resumir y comentar los estudios presentes en la literatura más relevantes y rigurosos sobre un determinado tema, con el objetivo de facilitar y agilizar la toma de decisiones. Este artículo se centra en la descripción del tipo de recursos y fuentes de información más significativas de la MBE, especialmente las accesibles a través de la Web que proporcionan información fiable.
\end{abstract}

Palabras clave: Medicina basada en la evidencia, Bases de datos, Fuentes de información, Literatura secundaria.

\section{Title: Information sources in evidence-based medicine}

Abstract: Evidence-based medicine has brought about a significant change in the role of information and its use on the part of health professionals, who must take appropriate decisions in their daily clinical practice. Evidence-based methodology has encouraged the creation of its own information sources that appraise, synthetise and provide comments on the most pertinent and rigorous current research on a given topic, with the goal of facilitating the decision-making process. The aim of this article is to describe evidence-based medicine's most relevant sources of information, especially those available over the Web that provide reliable answers to clinical questions.

Keywords: Evidence-based medicine, Databases, Information sources, Secondary literature.

\section{Introducción}

LAS RAÍCES de la medicina basada en la evidencia (MBE) se remontan a mediados del siglo XIX en París, con médicos como Louis, Bichat y Magendie, promotores de la "médicine d'observation", que sostenían que los médicos en su práctica asistencial no deberían basar sus decisiones únicamente en su experiencia personal sino también en los resultados de investigaciones que mostraran sus efectos en términos cuantificables (Sackett, 1996; Iambe, 1999). Estas ideas se rescataron a finales de la década de los 60 por un grupo de médicos, de los que cabe destacar David Sackett, Gordon H. Guyatt y Archibald Cochrane entre otros, que con la aplicación de principios de epidemiología clínica y de bioestadística se dieron cuenta de que podían encontrar respuestas apropiadas al tratamiento, diagnóstico y pronóstico de sus pacientes.
Estas nuevas ideas sobre el modo de practicar, enseñar y aprender la medicina se llegaron a conformar como un movimiento cultural internacional conocido como medicina basada en la evidencia, favorecido también por una serie de fenómenos propios de la revolución y explosión de la información, como la dificultad en el manejo del gran volumen de información científica médica para encontrar aquella más pertinente, los problemas para la actualización por parte de los médicos y el desarrollo de las nuevas tecnologías e internet. Este concepto moderno de MBE adquiere visibilidad ante el público profesional en 1992, cuando el grupo de trabajo de MBE, formado por internistas y epidemiólogos clínicos de la canadiense McMaster University, publica un artículo en la revista Journal of the American Association donde se propugna un nuevo paradigma para la práctica de la medicina, destacando la importancia de basar las decisiones clínicas en las evidencias halladas en la investiga- ción (Evidence Based Medicine Working Group, 1992).

En la actualidad es definida como el uso consciente, juicioso y explícito de la mejor evidencia actual para tomar decisiones sobre el cuidado del paciente individual. Su práctica consiste en la integración de la competencia clínica con la mejor evidencia clínica externa disponible a partir de la investigación sistemática (Sackett, 1996). Por mejor evidencia clínica externa disponible se entiende la investigación clínicamente relevante, especialmente la que se centra en el paciente y que estudia la exactitud y precisión de tests diagnósticos, la potencia de indicadores pronósticos así como la eficacia y seguridad de procedimientos terapéuticos, preventivos y rehabilitadores.

Su incorporación a la práctica clínica requiere cinco pasos (Sackett, 2001), de los que los dos primeros están relacionados directamente con el proceso de búsqueda de información: 
1. Definición de las preguntas clínicamente relevantes. Consiste en identificar la necesidad de información y traducirla al lenguaje de recuperación del sistema que se vaya a utilizar, es decir, diseñar la estrategia de búsqueda. Este paso no resulta fácil para el profano en la materia, pero es de gran importancia formular la pregunta de manera adecuada para obtener resultados relevantes y útiles. De ahí que en la literatura se recomiende el formato "PICO" para estructurar y precisar la pregunta, en el que se identifican hasta 4 elementos (Strauss, 1998; Centre for Clinical Effectiveness, 2005):

—población o pacientes, donde se describen las características más importantes como sexo y grupos de edad;

-intervención o indicador que interesa, como diagnóstico o tratamiento;

- comparación o control, si se desea comparar la intervención elegida con otra o con un grupo control; y

-resultado de interés (outco$m e$ ), por ejemplo: síntomas, exactitud del diagnóstico, mortalidad, etc.

2. Búsqueda de la mejor evidencia. Esta etapa consiste en la ejecución de la consulta en las fuentes de información apropiadas.

3. Valoración crítica de la evidencia. Una vez recuperada la información hay que determinar su validez y utilidad. Existe una amplia variedad de guías para valorar los diferentes tipos de artículos primarios publicados en revistas científicas, de las que se pueden destacar las publicadas en la revista $J A$ MA por el Evidence Based Medicine Working Group (1997).

4. Aplicar la evidencia a la práctica clínica. Una vez encontrada la información que se necesita, y determinada su validez, el siguiente paso consiste en integrar la eviden- cia aplicando los resultados a la situación clínica particular del paciente.

5. Evaluación del rendimiento de la MBE. Los profesionales sanitarios deben evaluar el funcionamiento correcto del proceso a través de la eficacia de los resultados obtenidos con la aplicación de la evidencia encontrada.

La MBE ha supuesto un cambio en el papel y en el uso de la información por parte del profesional de la salud que se ha enfrentado siempre, para tomar decisiones basadas en la mejor evidencia, a la gran dificultad de localizar y conseguir toda la información relevante, analizarla y sintetizarla. Proporcionar información de calidad que ya ha sido contrastada por expertos, en la que se analiza la validez metodológica de los estudios y se evalúa la relevancia clínica de los resultados (a la vez que se indican sus implicaciones en la práctica clínica diaria), viene a simplificar este proceso. En otras palabras, el médico recibe información sobre el mejor método que puede aplicar a un paciente particular.

\section{Tipos de publicaciones y jerarquía de la evidencia}

Para responder a las preguntas planteadas y obtener información precisa y fiable es necesario conocer los tipos de estudios que pueden dar la mejor respuesta. La MBE se nutre del conocimiento existente y buscar el que sea mejor es una tarea compleja que, a veces, resulta difícil ya que no todo lo que se publica tiene calidad y rigor metodológico. Haynes (2000) señala que sólo una pequeña parte de los estudios publicados superaría la prueba de una evaluación rigurosa con el suficiente margen para garantizar su aplicación clínica rutinaria.

La metodología basada en la evidencia ha favorecido la creación de recursos de información propios, entre los que destacan las revisiones sistemáticas y meta-análisis.

Las primeras examinan en profundidad la literatura médica con el objetivo de identificar y resumir toda la información relevante para formular la mejor aproximación a un diagnóstico o tratamiento. $\mathrm{La}$ elaboración de este tipo de estudio se realiza basándose en un protocolo estricto en el que se identifica, selecciona, valora y sintetiza la investigación relevante, además de recoger y analizar datos de los estudios que se incluyen en la revisión. Para minimizar el sesgo una revisión sistemática incluye o excluye la evidencia basándose en criterios de calidad explícitos.

Puede incluir o no meta-análisis, que son un tipo especial de revisión sistemática, en los que los estudios individuales deben cumplir unos criterios de homogeneidad entre sí. Además utiliza métodos estadísticos para analizar la literatura y dar una respuesta a una determinada pregunta clínica aumentando así la potencia de los resultados de numerosos estudios. El meta-análisis es una revisión en la cual se minimiza el sesgo por una reducción sistemática, síntesis y agregación estadística de todos los estudios relevantes de acuerdo con un método explícito y predeterminado (Cook, 1997).

Las revisiones sistemáticas se caracterizan por:

-título y objetivo de la revisión;

—estrategia de búsqueda utilizada para recuperar estudios relevantes que tratan los objetivos de la revisión;

-criterios explícitos y justificados de inclusión y exclusión de cualquier estudio;

- listado amplio de todos los estudios identificados; 


\section{LA NUEVA REVISTA SOBRE EL MUNDO DE LAS BIBLIOTECAS}

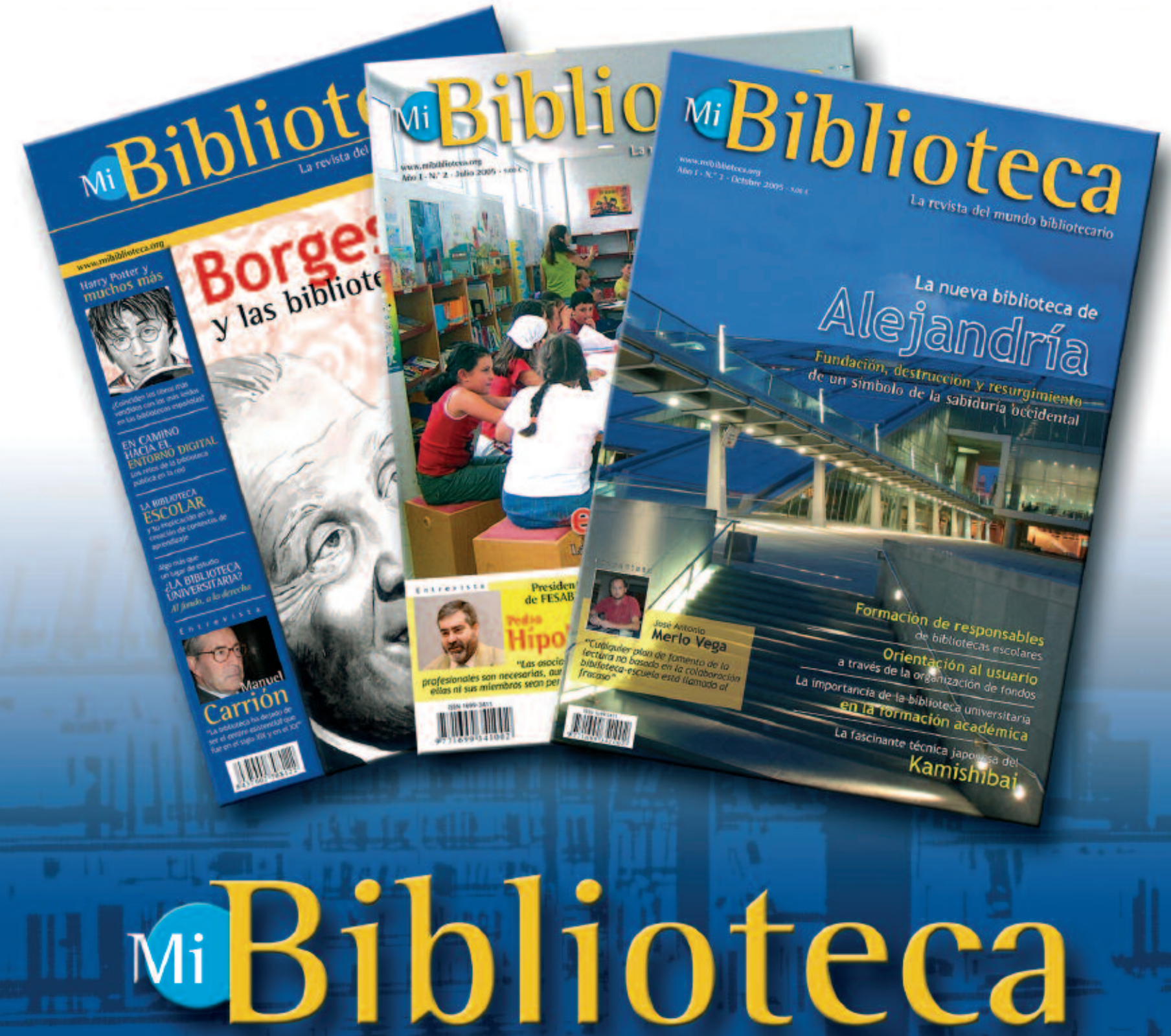

La revista del mundo bibliotecario

\section{Suscríbete.}


- listado de todos los estudios excluidos y justificación de su exclusión;

- presentación clara de las características de cada estudio incluido y un análisis de la calidad metodológica;

—análisis de los resultados de los estudios utilizando métodos estadísticos (meta-análisis), siempre que sea apropiado y posible; e

—informe estructurado de la revisión, con descripción de los objetivos, métodos y materiales, y presentación de los resultados.

Debido a que ningún estudio, independientemente de su tipo, debería de ser interpretado de manera aislada, una revisión sistemática es considerada, por lo general, la mejor forma de evidencia (Glasziou, 2004).

Con relación a los distintos tipos de estudios que se pueden encontrar en la literatura, se ha definido una jerarquía de la evidencia en diferentes niveles. Conforme se sube en el rango disminuye la cantidad de literatura disponible pero aumenta su relevancia desde el punto de vista clínico. Los niveles de evidencia reflejan el rigor metodológico de los estudios, situándose en el más alto los más rigurosos y menos susceptibles de sesgo. Aunque existen diversas clasificaciones de jerarquías propuestas por diferentes organismos, los niveles en la jerarquía de evidencia en cuanto al rigor del diseño de los estudios, tradicionalmente, han sido los siguientes (Harris, 2001):

-Ensayos controlados aleatorizados (nivel I).

-Ensayo controlado sin aleatorizar (nivel II-1).

-Estudios casos-control y de cohortes, preferiblemente multicéntricos o realizados por más de un grupo de investigación (nivel II-2).
-Múltiples series temporales con o sin intervención, o resultados espectaculares de experimentos no controlados (nivel II-3).

-Opiniones de expertos basadas en la experiencia clínica, estudios descriptivos e informes de casos, o informes de comités de expertos (nivel III).

\section{«La MBE ha supuesto un cambio en el papel y en el uso de la información por parte del profesional de la salud»}

En todos ellos son preferibles las revisiones sistemáticas o meta-análisis, siempre que se puedan aplicar. Esta escala de gradación de la evidencia es cierta cuando la pregunta a responder versa sobre el tratamiento, si bien no está libre de controversia y los propios promotores de la MBE afirman que no todas las preguntas sobre tratamiento requieren de un ensayo controlado aleatorizado (Sackett, 1997). Para otro tipo de preguntas pueden ser más adecuados otros diseños y tipos de estudios (Soto, 1998). De cualquier manera, el Third U. S. Preventive Services Task Force (Uspstf) hace hincapié en la importancia de valorar no sólo el tipo de estudio sino también la calidad, medida por la validez interna, y la calidad de toda la evidencia sobre el tema (Harris, 2001).

\section{Fuentes de información basada en la evidencia}

Durante los últimos años hemos asistido a un creciente interés por los recursos de síntesis y evaluación de información, lo que ha provocado un aumento importante de bases de datos y publicaciones especializadas en la materia. Todas ellas tienen una característica en común: que las revisiones y artículos que contienen se han sometido a un riguroso filtro de valoración crítica por parte de expertos que ase- guran la validez de la información que proporcionan. Este hecho ahorra al profesional médico el tercer paso del proceso de la MBE.

La búsqueda de soluciones basadas en la evidencia comienza con la selección de los recursos más apropiados, que son los que presentamos a continuación.

\section{Cochrane library}

Con tres bases de datos, fue creada por varios organismos, fundamentalmente el Centre for Reviews and Dissemination $(C R D)$ de la Universidad de York y la Colaboración Cochrane. Ésta, base de la MBE, es una organización mundial sin ánimo de lucro formada por expertos de 40 especialidades clínicas, agrupados en grupos colaboradores que se encargan de revisar las publicaciones, seleccionar las que cumplen los rigurosos criterios de MBE, realizar meta-análisis cuando es posible y escribir revisiones detalladas tanto del tema como de los resultados, siempre con el objetivo de proporcionar de manera rápida información fiable y actualizada sobre los efectos de la atención sanitaria.

La versión en castellano de la Cochrane Library es la Cochrane Library Plus, con acceso gratuito en todo el territorio español gracias a la suscripción realizada por el $M^{o}$ de Sanidad y Consumo, y está integrada por las siguientes bases de datos:

http://www.update-software.com/cl ibplus/clibplus.asp

- The Cochrane database of systematic reviews (CDSR). Contiene revisiones sistemáticas ya finalizadas o en proceso de realización (denominadas protocolos de revisión). Las revisiones tratan sobre la eficacia y conveniencia del tratamiento, así como otras intervenciones clínicas en determinadas enfermedades. Equipos de expertos se encargan de completar las revisiones de literatura, evaluando y sinte- 
tizando los resultados de la mejor evidencia. Periódicamente se añaden otras nuevas y se actualizan las ya existentes. Los protocolos incluyen una introducción, los objetivos y una sección de material y métodos de las revisiones en fase de preparación, con la fecha prevista de publicación.

-Database of abstracts of reviews of effects (DARE). Está creada y mantenida por el $C R D$ y se incluye bajo el enlace Other publisher reviews, aunque se puede acceder gratuitamente desde el sitio web del productor. Contiene resúmenes de revisiones sistemáticas que valoran la eficacia de los efectos o resultados de terapias, cubriendo temas no tratados en las revisiones de Cochrane. Sus responsables no realizan revisiones sistemáticas, sino que valoran críticamente las encontradas en las principales revistas de medicina, bases de datos bibliográficas, literatura gris y sitios web de calidad. De los artículos seleccionados se elabora un resumen sobre la eficacia de la intervención, las fortalezas y debilidades, seguido de un comentario crítico y sus implicaciones en la práctica clínica. Es un excelente complemento de la $C D S R$, ya que mientras la función de ésta es elaborar revisiones sistemáticas, DARE valora críticamente las realizadas por otros investigadores ajenos a la Colaboración Cochrane.

http://www.york.ac.uk/inst/crd/

-The Cochrane central register of controlled trials (Central). Contiene datos de ensayos controlados publicados en revistas indizadas en bases de datos bibliográficas, principalmente Medline y Embase, pero también de otras fuentes como comunicaciones a congresos. Incluye el título, datos bibliográficos y, en la mayoría de los casos, un resumen, pero no proporciona el texto completo.

- The NHS Economic evaluation database (NHS EED). Está producida por el $C R D$. Contiene resúmenes de artículos sobre evaluaciones económicas en atención sanitaria (análisis coste-beneficio, coste-eficacia y coste-utilidad). Los artículos se obtienen de bases de datos bibliográficas, revistas médicas importantes, etc., y se escogen los que comparan tratamientos y examinan los costes y resultados de las alternativas. Hay que destacar que tanto DARE como NHS EED proporcionan un comentario (valoración) sobre las investigaciones originales, pero no ofrecen el texto completo del artículo o documento original que valoran. Se puede acceder gratis desde la web del productor.

- The health technology assessment database (HTA). Creada por el $C R D$ en colaboración con la International Network of Agencies for Health Technology Assessment (Inahta), en la que España participa con 6 agencias. Contiene revisiones a texto completo, publicaciones breves y resúmenes sobre proyectos que se están llevando a cabo, y publicaciones finalizadas de organizaciones de evaluación de tecnología sanitaria. Las evaluaciones se refieren a prevención y rehabilitación, vacunas, productos y dispositivos farmacéuticos, procedimientos médicos y quirúrgicos, así como sistemas que protegen y mantienen el estado de la salud. No se evalúa la calidad de las publicaciones individuales. Es de acceso gratuito desde la web del productor.

- The Cochrane methodology register $(C M R)$. Es una bibliografía sobre métodos utilizados en los ensayos controlados. Contiene estudios de los procedimientos empleados en revisiones y en otros estudios metodológicos más generales que puedan ser relevantes para preparar revisiones sistemáticas.

Además de estas bases de datos, la Cochrane Library Plus contiene también, sólo en castellano, una base de datos de ensayos clínicos ibe- roamericanos y la traducción al castellano de la revista Bandolier.

\section{Bases de datos de guías de práctica clínica (GPC)}

Una GPC es un conjunto sistematizado de instrucciones que tiene como objetivo ayudar al médico y/o paciente en la adopción de las medidas más adecuadas ante un problema específico de salud. Constituyen otra fuente importante de evidencia evaluada, especialmente si están dirigidas al tratamiento o diagnóstico de una enfermedad. En los últimos años muchas asociaciones científicas, sociedades médicas, colegios profesionales, instituciones públicas de los sistemas nacionales de la salud y organizaciones privadas las han elaborado para diversas patologías, que se han recogido en bases de datos. Las GPC se basan en una amplia revisión bibliográfica y en una valoración crítica exhaustiva de los artículos recuperados.

Aunque no hay unos criterios claramente definidos para su elección, existen una serie de recomendaciones que conviene tener en cuenta (Cabana, 1999):

- Cumplir con los requisitos que la definen, es decir, que realmente sea una GPC y que esté basada en la evidencia.

-Que esté preferiblemente desarrollada bajo los auspicios de sociedades médicas, organismos gubernamentales, organizaciones públicas o privadas, y no por individuos o grupos de trabajo más o menos formalizados.

- Que se pueda comprobar que ha existido una búsqueda sistemática de la literatura y una revisión de la evidencia científica publicada durante el desarrollo de la guía, y que informe de la robustez y calidad de la evidencia en que se basa.

-Que se actualice periódicamente por parte de los autores, incorporando nuevas directrices o 
modificando las antiguas, según recomiende la nueva evidencia disponible.

Entre las bases de datos de guías clínicas, merece la pena destacar las de la U.S. National Guideline Clearinghouse, producida por la Agency for Health Care Policy and Research en asociación con la American Medical Association y la American Association of Health Plans; CMA Infobase de la Canadian Medical Association; NeLH Guidelines Database de la National electronic Library for Health del Reino Unido y Guidelines International Network (GIN).

http://www.guidelines.gov

http://mdm.ca/cpgsnew/cpgs/

index.asp

http://rms.nelh.nhs.uk/

guidelinesfinder

http://www.g-i-n.net/

En España cabe destacar las publicadas por el Centro Cochrane Iberoamericano y la sección que existe en Fisterra.

http://www.cochrane.es http://www.fisterra.com/recursos web/castellano/c_guias_clinicas.asp

\section{Bases de temas valorados críticamente}

Un critical appraisal topic (CAT) -término acuñado por el Center for Evidence Based Medici$n e$, de Oxford- es un resumen realizado por clínicos de una página de extensión de la evidencia disponible sobre preguntas clínicas comunes y según la metodología propuesta por la MBE. Un documento de este tipo responde a la siguiente estructura:

- título del tema valorado;

—pregunta clínica a responder;

—estrategia de búsqueda utilizada y bases de datos consultadas;

-artículos científicos que mejor responden a la pregunta;

-resumen de los resultados clínicamente importantes;
- comentarios sobre el diseño del estudio y su aplicabilidad, y

-en ocasiones, una bibliografía auxiliar que complementa los comentarios.

Son muchas las organizaciones, hospitales y centros médicos que han creado sus propios bancos de datos de CATs y los han puesto en la Red: CATBank del Center of Evidence-Based Medicine de Oxford; Evidence-Based Pediatrics Web Site de la Universidad de Michigan; UNC Critically Appraised Topics de la Universidad de Carolina del Norte; y BETs (Best Evidence Topics), del Departamento de Urgencias del Manchester Royal Infirmary del Reino Unido (sus registros son similares a los $C A T \mathrm{~s}$, pero modificados y adaptados a la medicina de urgencias).

http://www.cebm.net/cats.asp

http://www.med.umich.edu/pediatri cs/ebm/Cat.htm

http://www.med.unc.edu/medicine/ edursrc/!catlist.htm

http://www.bestbets.org/

«Sólo una pequeña
parte de los estudios
publicados superaría
la prueba de una
evaluación rigurosa
con el suficiente
margen para
garantizar su
aplicación clínica
rutinaria»

Una herramienta de gran utilidad es CAT Crawler, un metabuscador del Bionformatics Institute que utiliza 8 de las más importantes bases de datos de CATs.

http://www.bii.a-star.edu.sg/resear ch/mig/cat.asp

\section{Revistas}

Los artículos de las revistas científicas pasan un proceso de evaluación por miembros de su comité científico que determinan la calidad y conveniencia de su publicación.
A pesar de ello, se ha demostrado que buena parte de este material presenta defectos en el diseño, metodología o en la expresión de los resultados, lo que puede tener como consecuencia que el profesional de la salud incorpore información sesgada a su práctica clínica (Buñuel, 2003; McAlister, 1999).

Para superar estos inconvenientes, y como consecuencia del interés despertado por la $\mathrm{MBE}$, surge a principios de los 90 un nuevo tipo de publicación denominado revistas secundarias basadas en la evidencia, que publican $C A T \mathrm{~s}$, revisiones sistemáticas, meta-análisis y otros informes elaborados con métodos MBE.

De una manera general, y aunque con ligeras variaciones, la metodología para la publicación de trabajos en estas revistas suele ser la siguiente (Sackett, 2000):

a. Selección de revistas primarias con gran factor de impacto $\mathrm{y}$, por consiguiente, con alta probabilidad de ofrecer información relevante para la práctica del profesional de la salud.

b. Selección de los artículos de interés.

c. Valoración crítica de la validez del estudio.

d. Análisis por parte de médicos expertos en el tema de la relevancia clínica de los resultados que aparecen en los documentos que han superado el filtro metodológico.

e. Publicación del material que ha superado ambas cribas en un formato resumido, seguido de un comentario crítico en el que se analizan las posibles implicaciones de los resultados en la práctica clínica del profesional sanitario.

Han ido apareciendo revistas secundarias basadas en la evidencia en diferentes especialidades médicas: 
-ACP journal club. Es la más antigua y una de las más importantes sobre MBE en medicina interna. Publicada bimestralmente por el American College of Physicians y la American Society of Internal Medicine. Los editores examinan más de 100 revistas clínicas para identificar estudios rigurosos metodológicamente y clínicamente relevantes, proporcionando un resumen ampliado, conclusiones y un comentario sobre cada artículo seleccionado, además de recomendaciones clínicas. Requiere suscripción. http://www.acpjc.org/

-Evidence based medicine. Es una publicación bimestral del grupo editorial British Medical Journal, que selecciona estudios publicados en revistas médicas internacionales que tratan sobre necesidades y problemas clínicos concretos relacionados con la atención primaria. Requiere suscripción para acceder al texto completo aunque el acceso a la versión española es gratuito desde 2004.

http://ebm.bmjjournals.com/ http://ebm.isciii.es/

- Bandolier. Publicación mensual dirigida por el National Health Service británico que recoge y resume información médica basada en la evidencia. La versión on line es gratuita, aunque sus contenidos con respecto a la versión impresa tienen un retraso de entre 4 y 6 meses. Está traducida al español de manera desinteresada por un grupo de médicos y accesible a texto completo. http://www.jr2.ox.ac.uk/bandolier/ http://www.infodoctor.org/bandolera/

- Clinical evidence. Publicada por el grupo editorial de $B M J$, se actualiza mensualmente. Resume la mejor evidencia sobre intervenciones de más de 20 especialidades médicas y responde a cuestiones clínicas planteadas con frecuencia en las consultas.

http://www.clinicalevidence.com/ce web/conditions/index.jsp

El profesional de la información está abierto a todos los bibliotecarios, documentalistas y otros profesionales de la información, así como a las empresas y organizaciones del sector para que puedan exponer sus noticias, productos, servicios, experiencias y opiniones.

Dirigir todas las colaboraciones para publicar a: El profesional de la información

Apartado 32.280

08080 Barcelona

epi@elprofesionaldelainformacion.com

Hay que resaltar que en la actualidad son muchas las revistas médicas científicas que publican revisiones sistemáticas (JAMA, Annals of internal medicine, BMJ, Archives of internal medicine, The New England journal of medicine) y/o incorporan secciones dedicadas a la MBE como la BMJ o Journal of family practice, que incluyen en cada edición una sección de breves sumarios denominados Patientoriented evidence that matters (POEMs).

Como publicaciones especializadas en MBE originalmente publicadas en castellano, podemos destacar Evidentia, revista española de enfermería, y Evaluación de tecnologías sanitarias, gratis a texto completo y publicada desde el año 2002.

http://www.index-f.com/evidentia/i nicio.php

http://www.aeets.org

\section{Servicios de pregunta-res-} puesta

Hemos comentado anteriormente que en ocasiones no es fácil traducir la pregunta de manera apropiada y fácil para buscar en la literatura y obtener una respuesta. De ahí que para ayudar a los profesionales de la salud a resolver sus dudas ante un caso práctico, algunos servicios nacionales de salud han creado unidades especializadas en resolver las dudas mediante los servicios denominados de pregunta-respuesta.
Uno de los más conocidos es Attract (Ask trip to rapidly alleviate confused thoughts), creado por el $N H S$ británico, en el que un equipo de expertos responde a las preguntas enviadas por los profesionales sanitarios. Para ello, realizan la búsqueda bibliográfica en diversas fuentes y la valoración crítica, remitiendo al médico que envió la pregunta la mejor respuesta hallada o un resumen de los trabajos de investigación más relevantes en una cara de una página A4. Las respuestas se publican posteriormente en la web de esta organización, clasificadas por especialidades a través de un directorio temático, ordenadas por fechas, y se pueden consultar gratuitamente. Dispone de un buscador interno.

http://www.attract.wales.nhs.uk/

Otro recurso similar es ARIF (Aggresive research intelligence facility), de la Universidad de Birmingham, especializado en revisiones sistemáticas.

http://www.arif.bham.ac.uk/

La información de estos dos recursos se puede recuperar a través del motor de búsqueda TRIP, en su enlace Query-answering services.

Otro servicio es Family practice inquiries network (FPIN), consorcio estadounidense formado por departamentos de medicina de familia, redes de investigación en atención primaria, bibliotecas de ciencias de la salud y expertos individuales, que ofrece un servicio de respuestas en formato breve y que 
se publican también en la revista Journal of family practice.

Por último, citar el proyecto piloto Primary care question answering service, implantado por la $\mathrm{Na}$ tional Library for Health del NHS británico y dirigido a los profesionales de atención primaria.

http://www.fpin.org/

http://www.clinicalanswers.nhs.uk/

\section{Motores de búsqueda en la Web}

- TRIP (Turning Research Into Practice). Creado en 1997 por TRIP Database Ltd., es un metabuscador que utiliza simultáneamente 110 fuentes de información de gran calidad en la Web, incluida una búsqueda paralela en PubMed. Los resultados los muestra en una lista agrupados en las siguientes categorías: medicina basada en la evidencia, guías clínicas, servicios de pregunta-respuesta, libros de texto electrónicos, imágenes médicas, boletines de información para pacientes, artículos de revistas indizadas en Medline y BIG4 (revistas New England journal of medicine, JAMA, Lancet y British medical journal). Requiere suscripción, si bien permite cinco búsquedas gratuitas por semana para los no suscriptores.

http://www.tripdatabase.com/

-SumSearch. Metabuscador creado por la University of Texas Health Science Center, traducido y adaptado al español por Rafael Bravo, que realiza la consulta en varios motores de búsqueda como Dogpile, SavvySearch y Go2Net. Presenta la particularidad de que implementa el concepto de "búsqueda por contingencia" que consiste en que, según el número de resultados obtenidos, puede ampliarlos o reducirlos hasta conseguir un número óptimo. Los registros proceden en su mayoría PubMed, DARE y National Guideline Clearinghouse, por lo que suelen ser una mezcla de revisiones sistemáticas, guías y artículos originales. Los resultados se agrupan según el tipo de documento.

http://sumsearch.uthscsa.edu

http://sumsearch.uthscsa.edu/espan ol.htm

\section{Otros recursos en la Web}

-UpToDate. Es un programa educativo en el que colaboran las principales asociaciones médicas. Contiene revisiones de temas de medicina interna y subespecialidades, e integra la última evidencia con la experiencia clínica de los autores para proporcionar recomendaciones en el diagnóstico y tratamiento. Está escrito y editado por médicos expertos y se actualiza cada 4 meses.

http://www.uptodate.com

-PIER (Physician's Information and Education Resource). Publicada por el American College of Physicians, se actualiza trimestralmente y su acceso está restringido a los socios de ACP. Contiene recomendaciones prácticas sobre más de 400 enfermedades.

http://pier.acponline.org/index.html ?hp

-Poems (Patient oriented evidence that matters). Es una base de datos de artículos evaluados críticamente y que pertenecen, actualmente, a 106 revistas médicas. El material que presenta cubre temas de atención primaria, debiendo estar necesariamente orientados al paciente: mejora de síntomas, morbilidad, mortalidad y mejora de la calidad de vida. Se puede acceder a ella utilizando el sistema Inforetriever, que además también permite la búsqueda de resúmenes de las bases de datos de Cochrane, guías clínicas prácticas y otra información. http://www.infopoems.com/

\section{Bases de datos bibliográfi- cas de medicina}

Las razones para buscar también en este tipo de fuentes son diversas, pero pueden resumirse en que las revisiones existentes no se pueden generalizar a países o circunstancias específicas, es necesaria una actualización del tema de consulta, o puede que la pregunta no haya sido todavía cubierta en los recursos específicos basados en la evidencia (Bidwell, 2004). Por lo tanto, siguen desempeñando un papel importante, pero a diferencia de las especializadas en la MBE, es necesario tener en cuenta que los artículos recuperados en ellas deberán ser sometidos a un proceso de valoración crítica.

Son muchas las bases de datos existentes, y aunque por limitaciones de espacio no se pueden describir todas, caben destacar Embase y las producidas por la U.S. National Library of Medicine (NLM), especialmente Medline, accesible gratuitamente a través de PubMed. En esta última, desde Clinical queries se pueden recuperar fácilmente revisiones sistemáticas y estudios con rigor científico sobre el tratamiento y diagnóstico gracias a unos filtros de búsqueda (hedges) desarrollados por el Departamento de Epidemiología Clínica y Bioestadística de la McMaster University (Montori, 2005; Haynes, 2004; Haynes, 2005). Incluye búsquedas diseñadas para cuatro tipos de estudios en los que hay que especificar una intervención: tratamiento, diagnóstico, etiología y pronóstico.

De cualquier manera, para búsquedas complejas y que requieran varias ecuaciones de búsqueda, es recomendable realizar la consulta temática de manera habitual y posteriormente filtrar o limitar las citas recuperadas a los tipos de publicación que se deseen, como ensayos controlados aleatorizados, metaanálisis o al subconjunto de revisiones sistemáticas (la forma de escribir la consulta sería: systematic $[s b])$.

Otras bases de datos interesantes de la NLM son la Hstat (Health services/technology assessment 


\title{
Renovar (o comenzar) la suscripción a "El profesional de la información"
} es muy sencillo.

\author{
Usted puede gestionar online su suscripción conectándose a esta página \\ web:
}

\author{
http://www.elprofesionaldelainformacion.com/suscripciones.html \\ Si lo desea puede comunicar con nosotros dirigiéndose a esta \\ dirección de correo electrónico: \\ suscripciones@elprofesionaldelainformacion.com
}

text), que contiene el texto completo de revisiones sistemáticas, guías clínicas y evaluaciones de tecnología sanitaria; Hsrproj (Health services research projects in progress) y ClinicalTrials.gov, de ensayos clínicos.

\section{Bibliografía}

Bidwell, S. R. "Finding the evidence: resources and skills for locating information on clinical effectiveness". En: Singapore medical journal, 2004 , v. 45 , n. 12 , pp. $567-572$.

\section{Buñuel Álvarez, J. C.; González Rodríguez,} M. P. "Pediatría basada en la evidencia. Búsquedas bibliográficas a través de internet. Cómo encontrar la mejor evidencia disponible: bases de datos de medicina basada en la evidencia". En: Revista pediatría de atención primaria, 2003, v. V, n. 17, pp. 109-132.

Cabana M. D.; Rand, C. S.; Powe, N. R.; Wu, A. W.; Wilson, M. H.; Abboud, P. A.; Rubin, H. R. "Why don't physicians follow clinical practice guidelines? A framework for improvement". En: JAMA, 1999, v. 282, n. 15, pp. $1.458-1.465$.

Centre for Clinical Effectiveness, Monash Institute of Health Services Research, Melbourne, Australia. Evidence-based answers to clinical questions for busy clinicians, 2005. Consultado en: 13-07-05.

http://www.med.monash.edu.au/healthservices/c celebpcav10.pdf

Centre for Health Evidence. Users' guides to evidence-based practice. Consultado en: 10-07-05.

http://www.cche.net/usersguides/main.asp

Centre for Reviews and Dissemination, University of York, UK. Consultado en: 10-07-05.

http://www.york.ac.uk/inst/crd/index.htm

Cook, D. J.; Mulrow, C. D.; Haynes, B. R. "Systematic reviews: synthesis of best evidence for clinical decisions". En: Annals of Internal Medicine, 1997, v. 126, n. 5, pp. 376-380.

Evidence Based Medicine Working Group. "Evidence based-medicine. A new approach to teaching the practice of medicine". En: JAMA, 1992 , v. 268 , n. 17 , pp. $2.420-2.425$.
Evidence Based Medicine Working Group. "La medicina basada en la evidencia. Guías del usuario de la literatura médica". En: JAMA, Edición española, 1997, pp. 1-108.

Glasziou, P.; Vanderbroucke, J.; Chalmers, I. "Assessing the quality of research". En: $B M J$, 2004 , v. 328 , n. 7.340 , pp. 39-41.

Harris, R. P.; Helfand, M.; Woolf, S. H.; Lohr, K. N.; Mulrow, C. D.; Teutsch, S. M.; Atkins, D. "Current methods of the U. S. Preventive Services Task Force: a review of the process". En: American journal of preventive medicine, 2001, v. 20, n. 3 (supplement), pp. 21-35.

Haynes, R. B.; Gabriel Sánchez, R.; Jadad, A. R.; Browmaan, G. P.; Gómez de la Cámara, A. "Herramientas para la práctica de la medicina basada en la evidencia (I). Actualización en recursos de información basados en la evidencia para la práctica clínica". En: Medicina clínica (Barcelona), 2000, v. 115, n. 7, pp. 258-260.

Haynes, R. B.; McKibbon, K. A.; Wilczynski, N. L.; Walter, S. D.; Were, S. R. "Optimal search strategies for retrieving scientifically strong studies of treatment from Medline: analytical survey". En: $B M J, 2005$, v. 330, n. 7.501, pp. $1.179-1.184$.

Haynes, R. B.; Wilczynski, N. L. "Optimal search strategies for retrieving scientifically strong studies of diagnosis from Medline: analytical survey". En: $B M J, 2004$, v. 328 , n. 7.447, pp. $1.040-1.045$

Iambe. Instituto Argentino de Medicina Basada en las Evidencias. La medicina basada en evidencias. Consultado en: 24-10-05.

http://www.iambe.org.ar/que_es_mbe.htm

McAlister, F. A.; Clark, H. D.; Walraven, C.; Straus, S. E.; Lawson, F.; Moher, D.; Mulrow, C. D. "The medical review article revisited: has the science improved?". En: Annals of internal medicine, 1999, v. 131, n. 12, pp. 947-951.

Montori, V. M.; Wilczynski, N. L.; Morgan, D.; Haynes, R. B. "Optimal search strategies for retrieving systematic reviews from Medline: analytical survey". En: $B M J, 2005$, v. 330 , n. 7.482 , pp. 68-73.

Parada, A. "Información sobre ensayos clínicos". En: El profesional de la información, 1996, n. 49 (noviembre), pp. 13-14.
Rodríguez del Castillo Martín, M. «Conocimientos y capacidades del bibliotecario de ciencias de la salud en la actualidad». En: El profesional de la información, 2004, v. 13, n. 3 (mayo), pp. 191-196

Sackett D. L.; Straus, S. E.; Richardson, W. S.; Rosenberg, W.; Haynes, R. B. Medicina basada en la evidencia. Cómo practicar y enseñar la MBE, $2^{a}$ edición. Madrid: Harcourt, 2001, Isbn 84-8174-511-1.

Sackett, D. L.; Rosenberg, W. M.; Gray, J. A.; Haynes, R. B.; Richardson, W. S. "Evidence-based medicine: what it is and it isn't". En: $B M J, 1996$, v. 316, n. 7.023, pp. 71-72.

Sackett, D. L.; Wennberg, J. E. "Choosing the best research design for each question". En: $B M J, 1997$, v. 315 , n. 7.123 , pp. 1636.

Soto, J. "Medicina basada en la evidencia; pero en qué evidencia". En: Medicina clínica, 1998, v. 111, n. 14 , pp. 539-541.

Strauss, S. E.; Sackett, D. L. "Using research findings in clinical practice". En: BMJ, 1998, v. 317, n. 7.154, pp. 339-342.

José Antonio Salvador Oliván, profesor del Departamento de Ciencias de Documentación e Historia de la Ciencia de la Universidad de Zaragoza y responsable del Servicio de Información del Colegio de Médicos de Huesca.

jaso@unizar.es

José María Angós Ullate, técnico en información científica, Centro de Documentación Científica de la Universidad de Zaragoza.

angos@unizar.es

María Jesús Fernández Ruiz, responsable de la web del Ayuntamiento de Zaragoza.

mjferuiz@zaragoza.es

Rosario Arquero Avilés, Departamento de Biblioteconomía y Documentación, Universidad Complutense de Madrid. carquero@ccinf.ucm.es 